\title{
Travelling and standing envelope solitons in discrete non-linear cyclic structures
}

\author{
Aurelien Grolet ${ }^{1 *}$, Norbert Hoffmann ${ }^{1 \dagger}$, Fabrice Thouverez ${ }^{2 \ddagger}$ and \\ Christoph Schwingshackl ${ }^{1 \S}$ \\ ${ }^{1}$ Department of Mechanical Engineering, Imperial College London \\ ${ }^{2}$ Laboratoire de Tribologie et Dynamique des Systèmes, Ecole \\ Centrale de Lyon
}

\begin{abstract}
Envelope solitons are demonstrated to exist in non-linear discrete structures with cyclic symmetry. The analysis is based on the Non-Linear Schrodinger Equation for the weakly non-linear limit, and on numerical simulation of the fully non-linear equations for larger amplitudes. Envelope solitons exist for parameters in which the wave equation is focussing and they have the form of shape-conserving wave packages propagating roughly with group velocity. For the limit of maximum wave number, where the group velocity vanishes, standing wave packages result and can be linked via a bifurcation to the non-localised non-linear normal modes. Numerical applications are carried out on a simple discrete system with cyclic symmetry which can be seen as an reduced model of a bladed disk.
\end{abstract}

\section{Nomenclature}

$N_{s} \quad$ total number of sector

$a \quad$ sector parameter $\left(a=2 \pi / N_{s}\right)[\mathrm{rad}]$

$u_{n} \quad$ displacement of sector $n[\mathrm{~m}]$

$m_{s} \quad$ mass of each sector $[\mathrm{kg}]$

$k_{s} \quad$ on-site linear stiffness $\left[\mathrm{kg} . \mathrm{s}^{-2}\right]$

\footnotetext{
*a.grolet@imperial.ac.uk, adress all corespondance to this author

†n.hoffmann@imperial.ac.uk

‡fabrice.thouverez@ec-lyon.fr

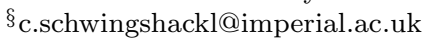




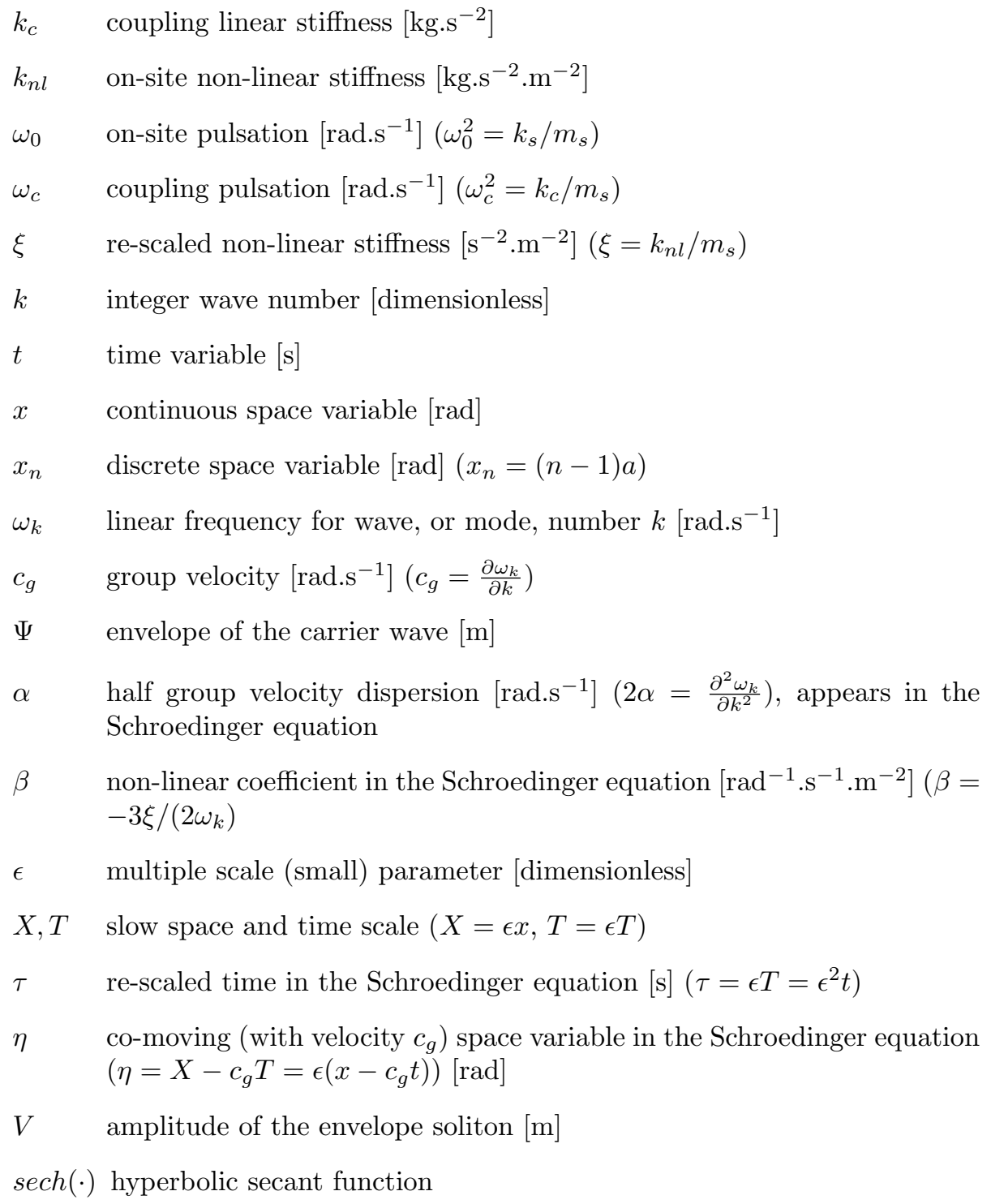

\section{Introduction}

Cyclic structures are of eminent relevance for a large number of disciplines in mechanical and civil engineering. Modern large scale buildings are often composed of modular, repeating elements. Rotating machinery almost always contains components with some sort of cyclic symmetry, for example bladed disks (blisks) of compressors and turbines in turbo-machinery. This study has been motivated most strongly from aeronautical engineering, where cyclic structures are of particular interest in aero-engines. The pressing need to further reduce 
weight leads to a number of design imperatives, like reduced clearances between rotating and stationary components or the introduction of new light-weight materials. In sum, non-linear effects, arising from material or geometric nonlinearities, have to be taken into account more and more accurately in order to model the system behaviour adequately, especially when vibration is concerned. Within the the field, localisation phenomena are of particular interest and growing importance.

For linear systems it is well known that deviations from homogeneity or symmetry may lead to localisation of vibration amplitude. The effect has first been observed in solid state physics and is there known as Anderson localisation [10]. Later in turbo-machinery the effect has been re-discovered and is now, with a view to the spectral perspective, most widely known as mistuning $[2,13]$. In the homogeneous case, there is a perfect discrete rotational symmetry, which means that each segment of the structure is mechanically identical. Then the vibration modes are doublets and can be viewed as two spatially phase-shifted stationary waves or two travelling waves with opposite velocities. If the discrete rotational symmetry of the system is slightly broken, one then often talks about a detuned system, the double modes split in frequency and the localisation phenomenon appears (see eg. $[2,28]$ ): spatial vibration localisation may then range from only slight inhomogeneity in the response, up to complete spatial confinement, where only a small subset of the sectors vibrates with large amplitude, while the other sectors remain virtually motionless.

This mechanism of vibration localisation, based on slight inhomogeneity, or symmetry breaking, of the otherwise linear system, very often forms the fundamental starting point for analysis into vibration response and fatigue of many components and sub-systems in turbo-machinery. This is quite a remarkable aspect, taking into account that today the components under consideration are subjected to higher and higher loads, experience large displacements under normal operational and extreme loading conditions in failure cases.

For non-linear systems in general, localisation may occur in perfectly tuned systems, where the structural properties are spatially homogeneous, or at least invariant under a discrete symmetry transformation. There seems to be substantial debate on mechanisms underlying localisation, e.g. if there is only a single dominant localisation mechanism, in terms of mathematics or in terms of mechanics, or if there are several independent ones: while Anderson localisation or mistuning induced localisation is a linear mechanism, in other fields of physics non-linear localisation mechanisms are prevalent. For example, nonlinear localisation may arise due to the dependence of the vibration mode shape on amplitude ('non-similar modes') [5,26]. Localisation may also occur through bifurcations from the main normal modes branches [5,6,21]. Those localized stationary solutions are often referred to as Intrinsic Localized Modes (ILM) or Discrete Breathers (DB) [4,25], and can be computed directly with numerical methods such as the Harmonic Balance Method (HBM) coupled with polynomial system solving methods (i.e. homotopy [21], or Groebner basis [7]). In contrast to such stationary solutions, travelling waves can also be computed us- 
ing the HBM, and it has been shown that such solutions can also bifurcate into localized travelling waves solutions (see eg [6]). Localised motion has also been observed numerically in various granular chains taking into account Hertzian contact theory (see eg. [24]).

Remarkably, most of the available work on non-linear vibration localisation in structural dynamics seems to be largely unrelated to the work done in many of the physics disciplines concerned with non-linear waves. While solitary waves, solitons, breathers etc have now been studied for decades in a large number of fields in physics, and have led to ground-breaking insight and to numerous applications, there seems to be a gap between the physics oriented disciplines and the engineering oriented disciplines. This is particularly true for structural dynamics, especially applied to mechanical engineering, or in our application case turbo-machinery, where there is only a very small number of studies about the numerical or experimental generation of solitons. Most of the studies focus on systems such as discrete lattices (e.g. [1,9,18]) or granular chains (e.g. [24]). Only a few studies are dealing with with solitary waves in continuous structure, see e.g. [14-17]. The reasons underlying this scarcity of work on solitons in structural dynamics are many-fold. The main reason probably being that the deliberate generation of solitons in structures seems to be more difficult than in other media: while for many systems of physics (water waves, non-linear optics, plasma physics etc.) the non-linear terms in the evolution equations are well known and easy to identify, for systems of solid mechanics analytical approaches are more involved, and only the advent of today's computing techniques seems to make inclusion of non-linearities a manageable and pragmatic affair.

While the importance and significance of homogeneous travelling wave modes is fully acknowledged in the field of turbo machinery, the potential role of finite-amplitude localised wave-packages does not seem to have been considered in the community. However, experimental studies such as [16] have shown that solitary waves can indeed appear in thin continuous rotating structure, possibly through fluid structure interaction. The main ingredients for solitons (dispersion, non-linearities) along with some particular features (cyclic symmetry, fluid structure interaction, ...) are also present in turbo-machinery, which make us think that solitary waves could also happen for example in a bladed disk. Taking into account such localized travelling waves could be useful for design strategy, in particular in order to avoid failure when localized vibration happens. Another application of solitary wave could concern default detection in bladed disks, by studying the discrepancy between theoretical an experiential solitary waves, as it is done for example for hyper-elastic elastic rods in [14].

The objective of this paper is to contribute to bridging the gap in the knowledge on soliton like states between physics and mechanical engineering. Here we will particularly focus on envelope solitons $[9,18]$ in the context of nonlinear structures with cyclic symmetry, which are of particular importance for turbo-machinery. We will show the existence of weakly and strongly non-linear 
envelope soliton solutions in a simple non-linear cyclic structure with discrete elements, which can be seen as a simplified model for a bladed disk structure. Using the Non-Linear Schrodinger Equation and its envelope soliton solutions as a starting point, both travelling and standing solitary vibration states are derived.

The paper is organised as follows. First we introduce our model and the techniques needed to derive envelope soliton solutions for it. We then apply the techniques and illustrate the existence of travelling envelope solitons for different parameter sets. In the following section we demonstrate the existence of standing soliton solutions and show how they can be understood to arise through bifurcations from the well known non-linear non-localised modal solutions. Finally we conclude with a summary, critical remarks, and some potential perspectives.

\section{Modelling and solution approach}

\subsection{The model}

We consider an oscillator chain with local cubic non-linearity, basically a series of linearly coupled Duffing oscillators, as depicted in Fig.1. The equations of

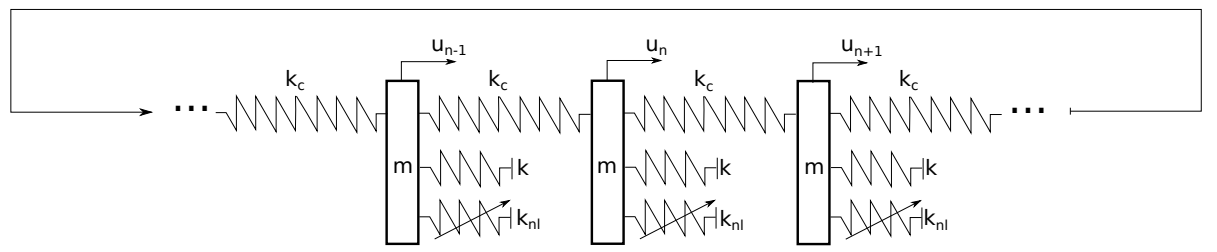

Figure 1: Schematic representation of the cyclic system studied in this paper.

motion for such a system have the following form $\left(n \in\left[1, N_{s}\right]\right)$

$$
m_{s} \ddot{u}_{n}+\left(k_{s}+2 k_{c}\right) u_{n}-k_{c}\left(u_{n-1}+u_{n+1}\right)+k_{n l} u_{n}^{3}=0 .
$$

The system can be seen as a simple idealised model for a bladed disk structure composed of $N_{s}$ sectors where $u_{n}$ stands for the displacement of the $n^{\text {th }}$ blade $[5,6]$. Introducing the sector parameter $a=\frac{2 \pi}{N_{s}}$, Eq.(1) can be rewritten in the following form:

$$
\ddot{u}_{n}+\omega_{0}^{2} u_{n}+\xi u_{n}^{3}=\omega_{c}^{2}\left(u_{n-1}+u_{n+1}-2 u_{n}\right),
$$

with $\omega_{0}^{2}=\frac{k_{s}}{m_{s}}, \omega_{c}^{2}=\frac{k_{c}}{m_{s}}, \xi=\frac{k_{n l}}{m_{s}}$. 


\subsection{Linear analysis}

Here we consider the underlying linear system of Eq.(2) given by the following:

$$
\ddot{u}_{n}+\omega_{0}^{2} u_{n}=\omega_{c}^{2}\left(u_{n-1}+u_{n+1}-2 u_{n}\right) .
$$

Substituting the cyclic condition for the wave number $k$ (i.e. $u_{n}^{(k)}(t)=$ $U_{k} e^{i\left[k(n-1) a-\omega_{k} t\right]}+$ c.c., where c.c. denotes the complex conjugate) into Eq.(3) leads to the dispersion relation $\left(\omega_{k}\right.$ is the frequency of the wave with wave number $k$ ),

$$
w_{k}^{2}=\omega_{0}^{2}+2 \omega_{c}^{2}(1-\cos (k a)),
$$

and the mode shapes:

$$
\phi_{k}=\mu_{k}\left[1, e^{i k a}, \ldots, e^{i k\left(N_{s}-1\right) a}\right],
$$

where $\mu_{k}$ is a factor to keep the mode shapes scaled relative to the mass matrix $\left(\mu_{0}=1 / \sqrt{N_{s}}, \mu_{N_{s} / 2}=-1 / \sqrt{N_{s}}, \mu_{k}=\sqrt{2 / N_{s}}\right)$.

From Eq.(4) it can be seen that the discrete linear angular frequencies lie within the bounded range $\left[\omega_{0}, \sqrt{\omega_{0}^{2}+4 \omega_{c}^{2}}\right]$ where the upper bound is due to the discreteness of the system. Using the dispersion relation in Eq.(4) we can define two quantities that will prove to be useful later on: (i) the group velocity $c_{g}=\frac{d w_{k}}{d k}$ and (ii) the group velocity dispersion $G V D=2 \alpha=\frac{d^{2} w_{k}}{d k^{2}}$, given by

$$
c_{g}=\frac{d w_{k}}{d k}=\frac{a \omega_{c}^{2}}{w_{k}} \sin (k a), \quad 2 \alpha=\frac{d^{2} w_{k}}{d k^{2}}=\frac{1}{w_{k}}\left(a^{2} \omega_{c}^{2} \cos (k a)-c_{g}^{2}\right)
$$

\subsection{Derivation of envelope soliton solutions}

We now derive envelope soliton solutions for our system, compare e.g. [18]. The solutions are supposed to have the form of a modulated carrier wave,

$$
u_{n}(t)=\Psi_{n} e^{i\left(k(n-1) a-\omega_{k} t\right)}+\text { c.c. },
$$

where c.c. denotes the complex conjugate, $k$ the wave-number, and $\Psi_{n}=$ $\Psi(n a)=\Psi(x)$ the envelope. Using the continuum approximation $(a<<1)$, the amplitude of the next sector can be expressed using Taylor expansion:

$$
\Psi_{n \pm 1}=\Psi(n a \pm a)=\Psi(x) \pm a \frac{d \Psi}{d x}(x)+\frac{a^{2}}{2} \frac{d^{2} \Psi}{d x^{2}}(x)+\text { H.O.T. }
$$

(where H.O.T. stands for High Order Terms).

Furthermore, we assume that the envelope varies slowly in time and space (as compared to the carrier wave) by introducing slow time and space scales $T=\epsilon t$ and $X=\epsilon x$ where $\epsilon$ is a small parameter, leading to the following form for the soliton solution:

$$
u_{n}=\Psi(X, T) e^{i\left(k(n-1) a-\omega_{k} t\right)}+\text { c.c. }=2 \operatorname{Re}\left(\Psi(X, T) e^{i\left(k(n-1) a-w_{k} t\right)}\right)
$$


Using the ansatz (9) together with the continuum approximation (8), it can be shown (appendix A) that the envelope should follow a Non-Linear Schrodinger Equation (NLSE):

$$
i \frac{\partial \Psi}{\partial \tau}+\alpha \frac{\partial^{2} \Psi}{\partial \eta^{2}}+\beta|\Psi|^{2} \Psi=0,
$$

with $\tau=\epsilon T, \eta=X-c_{g} T, \alpha=\frac{1}{2} \frac{d^{2} w_{k}}{d k^{2}}, \beta=-\frac{3 \xi}{2 \omega_{k}}$, and where the group velocity $c_{g}$ and group velocity dispersion $\alpha$ have been defined in Eq.(6).

There is a wide variety of solutions already known for the NLSE, see e.g. [8], and the derivation of new solutions is still an active field of research in applied mathematics.

Using the particular ansatz $\Psi(\eta, \tau)=a(\eta) e^{i \gamma \tau}$, it can be shown that the square of the amplitude $A(\eta)=a(\eta)^{2}$ should follow the following differential equation :

$$
\frac{\alpha}{2 \beta}\left(\frac{\partial A}{\partial \eta}\right)^{2}=-A^{3}+\frac{2 \gamma}{\beta} A^{2}+\frac{4 E}{\beta} A=-A\left(A-A_{2}\right)\left(A-A_{3}\right)=P(A)
$$

where $E$ is an integration constants, and $A_{2}, A_{3}$ are the roots of the polynomial $X \mapsto X^{2}-\frac{2 \gamma}{\beta} X-\frac{4 E}{\beta}$.

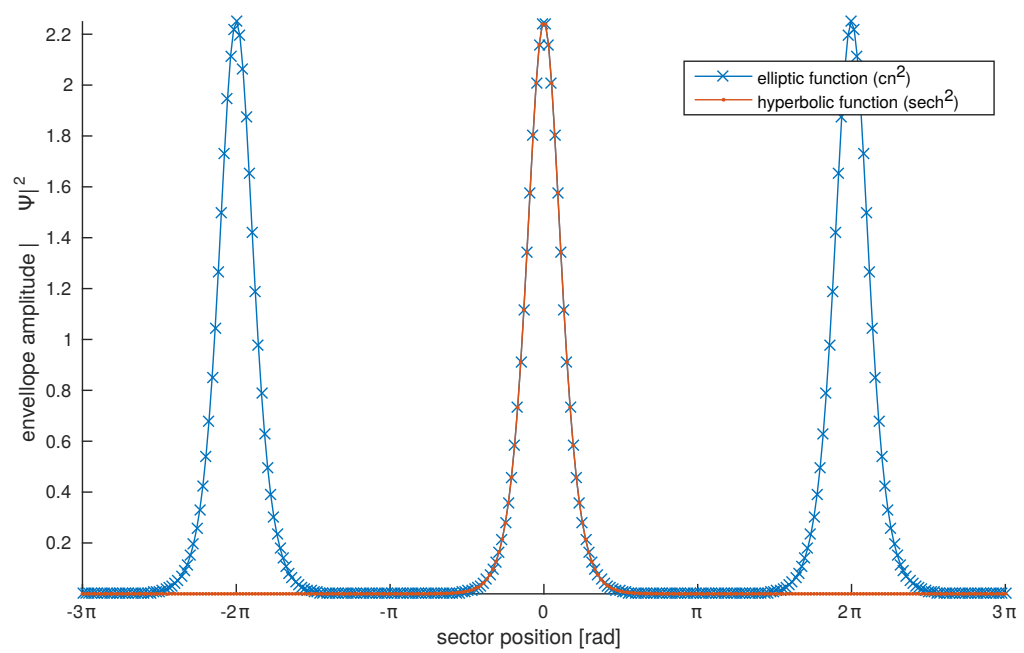

Figure 2: Example of comparison between elliptic and hyperbolic functions for the representation of localized solutions of (10) (square of the amplitude has been ploted).

In this paper we focus on envelope solitons which are known to exist for $\frac{\alpha}{\beta} \geq 0$, what is sometimes called the focussing range. The general solution of (11) involves elliptic functions (see for example [18]). However when the solution is highly localized, the (elliptic) solution can be well approximated 
over one period by a hyperbolic secant function (see Fig.2). The approximated solutions then have the following form:

$$
\Psi(\eta, \tau)=V \operatorname{sech}\left(V \sqrt{\frac{\beta}{2 \alpha}} \eta\right) e^{i \frac{\beta}{2} V^{2} \tau},
$$

where $\operatorname{sech}(\cdot)$ denotes the hyperbolic secant, defined as the inverse of the hyperbolic cosine. Going back to physical coordinates and dropping $\epsilon$ gives the following expression for the envelope soliton:

$$
u_{n}(t)=2 V \operatorname{sech}\left[V \sqrt{\frac{\beta}{2 \alpha}}\left((n-1) a-c_{g} t\right)\right] \cos \left[k(n-1) a-\omega_{k} t+\frac{\beta}{2} V^{2} t\right] .
$$

This solution corresponds to a travelling wave, the carrier, modulated by an envelope in the form of a hyperbolic secant propagating with group velocity $c_{g}$. Note that the only free parameter in the solution is the amplitude $V$, and that the resulting wave packet becomes more and more localised when the amplitude $V$ increases. In our case, the non-linear coefficient $\beta$ is always negative, so the condition $\frac{\alpha}{\beta} \geq 0$ indicates that an envelope soliton will only be possible for a sufficiently high wave number $k$, i.e. a short enough carrier wavelength. Finally, we can see that the phase of the solution is adjusted to take into account the cubic non-linearity, leading to an effective angular frequency given by

$$
\omega=\omega_{k}-\frac{\beta}{2} V^{2}=\omega_{k}+\frac{3 \xi}{4 \omega_{k}} V^{2} .
$$

As a result of this analysis, by using the expression in Eq.(13) one is able to compute initial conditions for generating soliton like solutions in the oscillator chain, or cyclic lattice, under study.

\section{Numerical analysis of envelope soliton solu- tions}

\subsection{Parameters}

In the following we consider a simple example of an oscillator chain, or cyclic lattice, depicted on Fig.1 and governed by Eq.(1) with the following numerical values for the parameters:

$$
N_{s}=100, \omega_{0}^{2}=1, \omega_{c}^{2}=1, \xi=0.1, a=\frac{2 \pi}{N_{s}} \approx 0.063 .
$$

The overall behaviour of the angular frequency Eq.(4), the group velocity and group velocity dispersion (GVD) Eq.(6), as a function of the wave number $k$ is shown in Fig.3. One can see that the system has two regimes: positive GVD and negative GVD. Fig.3 also shows the evolution of the non-linear coefficient $\beta$ as a function of the wave number. As noted earlier, we will focus on envelope solitons which are expected to appear in the focussing range, i.e. for $k \in[18,50]$ (i.e. for $\frac{\alpha}{\beta} \geq 0$ ). 

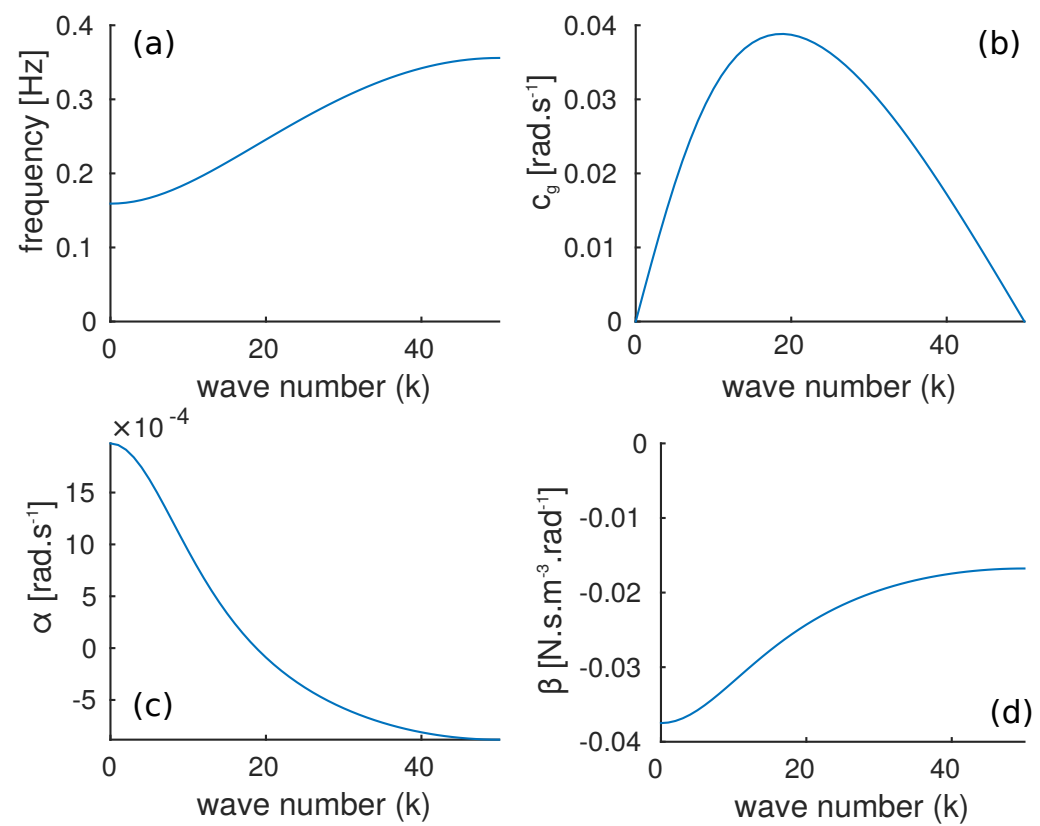

Figure 3: Evolution of the frequency (a), group velocity (b), group velocity dispersion (c) and non-linear coefficient (d) as a function of the wave number $k$ for the simple cyclic lattice of Eq.(1).

\subsection{Numerical analysis}

Using the above derived analytic expressions and Eq.(13) it is possible to derive initial conditions (positions and velocities) to generate envelope soliton through the use of classical temporal integration schemes applied to Eq.(2).

Simulations have been carried out for a number of different characteristic wave numbers and various amplitude levels. Results for two different wave numbers are shown in Fig.4 $(k=25)$ and Fig.5 $(k=40)$. The first row of the figures depicts the time evolution of the solutions' envelopes in a reference frame moving with group velocity (i.e as a function of $\eta=x-c_{g} t$ ). The envelope functions are computed using the Hilbert transform [3]. The second row of the plots shows the time evolution of an arbitrary degree of freedom (the first one was selected here) for the full non-linear system, while the third row shows the time evolution of the linearised system (in which the cubic non-linearity has been dropped). The three columns corresponds to different amplitude values of the solitons.

A number of results can be observed from the figures. First, we discuss what happens when the amplitude of the soliton is varied. For low amplitude, the envelope extends over the whole domain. It is thus not strictly speaking 

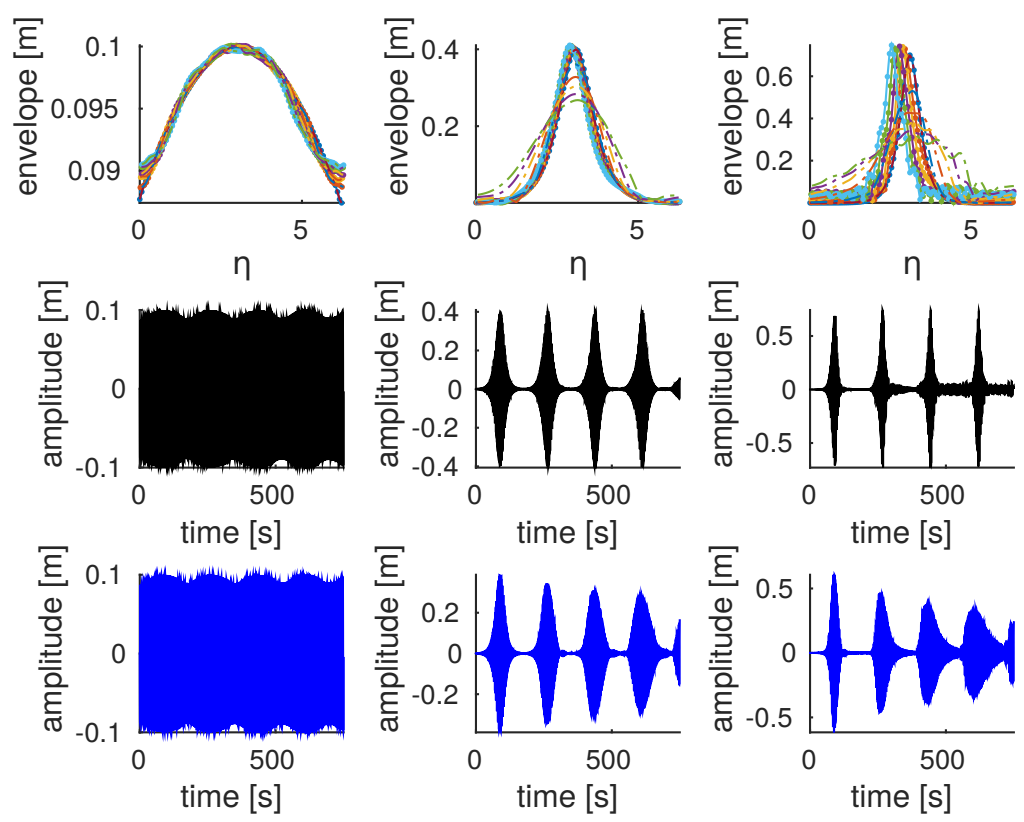

Figure 4: Numerical evolution of soliton solutions for carrier wavenumber $k_{c}=$ 25 and soliton amplitudes $V=0.1,1,5$ (left to right). Top row: evolution of the envelope over time with $\Delta t=150 \mathrm{~s}$ (-: non-linear solution, $-\cdot-$ : linear solution), middle and bottom row: temporal evolution of the non-linear and the linearised system.

spatially localized in the usual definition. With a view to the finite size of the system at hand, and compared to the infinite system from which the original soliton solution was taken, this reveals that the width of the packet is too large to fit in full into our system. Instead there is only an amplitude modulation of the envelope over the domain. The non-linear results are very similar to the linear ones, at least on the time-scales under discussion here.

For larger amplitudes, the width of the envelopes is smaller and the wave packets do fit in full into the domain. Observing the packets from a stationary observer, they seem to pass by again and again. However, one can now also clearly see the effect of dispersion on the linear system: the envelope broadens strongly with time. In the non-linear case, the velocity of each frequency component also depends on its amplitude, so that the differences in velocity for each wave number are compensated by the non-linearity. As a result, the envelope does not change shape during propagation and is actually travelling with group velocity $c_{g}$. That is why the envelopes at different times almost coincide in the co-moving frame. All these results are a striking confirmation of soliton type 

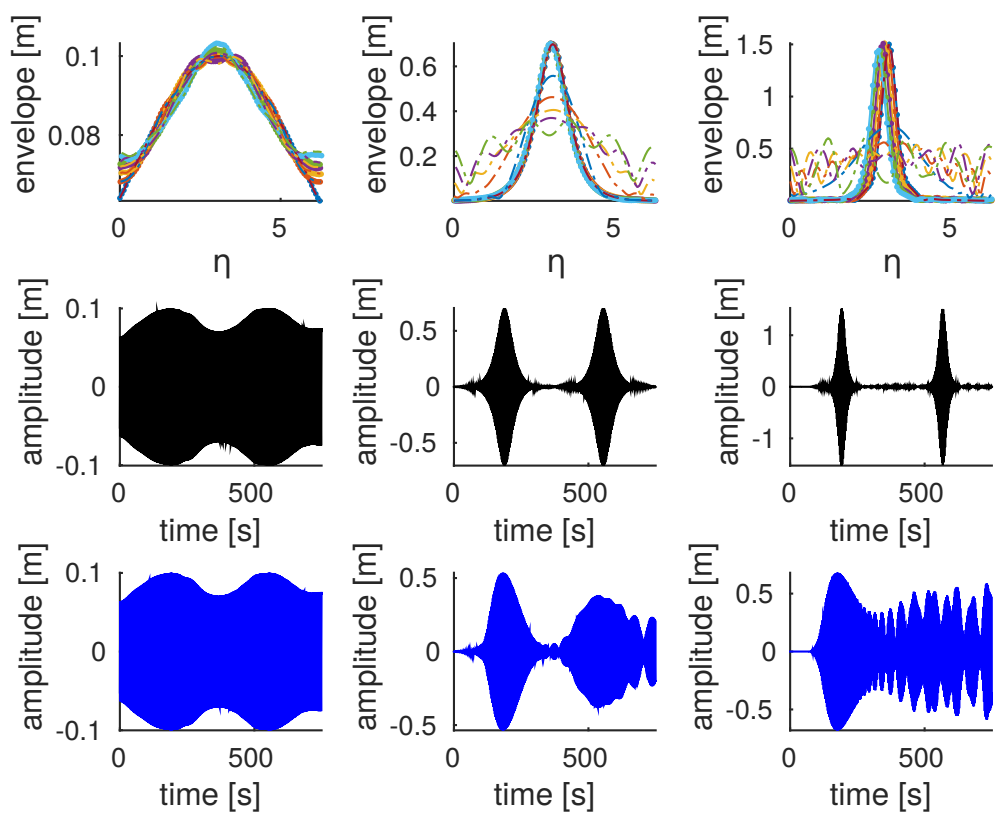

Figure 5: Numerical evolution of soliton solutions for carrier wavenumber $k_{c}=$ 40 and soliton amplitudes $V=0.1,0.7,1.5$ (left to right). Top row: evolution of the envelope over time with $\Delta t=150 \mathrm{~s}$ (-: non-linear solution, $-\cdot-$ : linear solution), middle and bottom row: temporal evolution of the non-linear and the linearised system.

dynamics.

For even larger amplitude, where the width of the packets is even smaller, we can see that the shape of the wave packets remains rather well approximated by the envelope soliton in the sense that the envelope does not change over time. However, we can also see that the envelopes do not exactly coincide in the moving frame, indicating that the velocity of the envelope is slightly different from the group velocity. This is an expected property for stronger non-linearity, since the solutions of the NLSE are strictly speaking valid only for asymptotically weak non-linearity.

\subsection{Remarks}

The envelope solitons presented in the previous section can be considered quasi periodic solutions of the system, since the group velocity is a priori not commensurable with the carrier wave velocity, and can be compared to non-linear normal modes (NNM). NNM are classically defined as solutions of the unforcedundamped equation of motion where each dof vibrates at unison (Rosenberg 
definition $[19,20])$. Alternatively, they can be defined as a manifold such that if the initial conditions are taken at any point of the manifold, the resulting motion will remains on the manifold for all times (invariant manifold definition $[22,23])$. In particular, this wider definition allows a travelling wave to be a NNM. From the previous results, we saw that solitons are solution of the undamped, unforced system and if a solution is initiated in the form of a soliton, then it remains in this form for all time (assuming the solitons are stable). In this context, solitons have many similarities with NNM and might be considered as an extension of the concept.

\section{The limit of maximum carrier wave-number}

Up to now we have only considered cases with carrier wave-numbers from the inner domain of the focussing zone of the dispersion relation. In our system, however, the focussing zone has two boundaries: The lower boundary where the focussing system changes over to the defocussing system. We will not consider this limit here. The upper boundary corresponds to the maximum carrier wavenumber possible in the system.

Here we should remark, that fully analogous results have been found in the context fo solid state physics [4] a while ago. There this case is called the band-gap limit, and the resulting localised modes, which now are usually called ILMs in that context, had a substantial impact on the field. Although formally much of the following discussion could be translated from the physics context, we still think a discussion in the present context of mechanical engineering and turbo-machinery might be useful to these disciplines.

In the case where the number of sectors is even, the carrier wave with maximum wavenumber simply corresponds to each of the neigbouring sectors moving exactly out of phase. This is a very interesting limit, since it will turn out in the following that the corresponding envelope solitons actually do not travel, and moreover they can be linked via a bifurcation with the spatially homogeneous time-periodic non-linear normal modes.

\subsection{Standing solitons}

When the number of sectors is even, there exists a mode where consecutive sectors vibrate out of phase. This mode corresponds to the wave number $k=$ $N_{s} / 2$. Its linear angular frequency is given by $\omega_{N_{s} / 2}^{2}=\omega_{0}^{2}+4 \omega_{c}^{2}$ and its shape is of the form $\phi_{n}^{\left(N_{s} / 2\right)}=(-1)^{n}$. This mode is actually not travelling and can be seen as a standing wave. Moreover, the group velocity vanishes for this particular mode (Eq.(6)). Consequently, the soliton solution given by Eq.(13) corresponds to a standing wave packet which is spatially more and more localized (almost up to a single sector) as the amplitude increases (see Fig.6-7). Even though the solution is not travelling any more, we will still be referring to it as a soliton. 
Using Eq.(13) initial conditions can be derived to initialise a soliton solution in the case of mode $N_{s} / 2$. Fig. 6 shows the comparison between linear and non-linear solutions for different amplitudes. For low amplitude, both linear and non-linear solutions give qualitatively the same results. For medium and large amplitudes, we can see that the envelope of the linear solution is distorted over time (due to the dispersion effects), whereas the envelope of the non-linear solution tends to keep a constant shape. Note that in this case, however, the envelopes of the non-linear solutions at different times do not strictly coincide due to the fact that the solution is now breathing (i.e. is of the form $u(x, t)=$ $\Psi(x) \cos (\omega t))$, so that at each time $t_{m}$ the envelope is multiplied by a factor $\cos \left(\omega t_{m}\right)<1$.
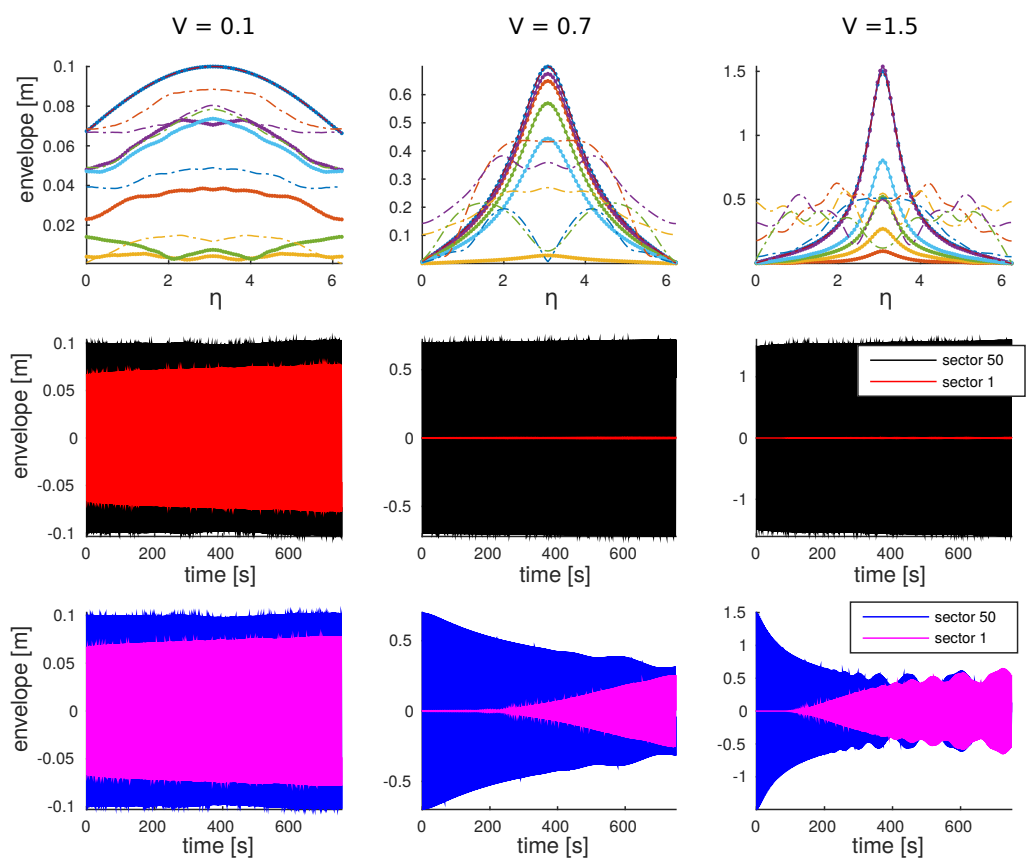

Figure 6: Numerical example of soliton solutions of system (2) for $k_{c}=50$ and for various amplitudes (left to right $V=1,5,10$ ). Row 1: evolution of the envelope at different time with $\Delta t=150 \mathrm{~s}$ (-: nonlinear solution, $-\cdot-$ : linear solution). Row two and three: temporal evolution of the non-linear and the linear system.

One should note here that this finding is quite remarkable in the sense that the NLSE should strictly speaking not be applicable for this limit case, since the underlying expansion approach that is applied in its derivation requires weak variations of the envelope. On the contrary, for this high wave-number limit the envelopes can hardly be understood any more as weak modulations of an un- 
derlying carrier. Surprisingly, the numerical results do suggest that initialising the fully non-linear calculations with initial conditions from the NLSE envelope solitons yields soliton like solutions via time-integration. To elucidate the behaviour, we turn to further numerical analysis that will be shown to confirm this finding.

\subsection{Harmonic balance method, continuation and bifurca- tions}

The Harmonic Balance Method (HBM) is a wide-spread technique to find periodic solution of non-linear dynamic systems via truncated Fourier series [7,27]. The aim of this section is to compare the standing soliton solutions as obtained from numerical simulation with results obtained from applying the HBM in the case of mode $N_{s} / 2$.

Using only a single time-harmonic approximation for the displacement, the HBM solution is searched for under the following form (monophase NNM [5], where here a factor of 2 is introduced for easy comparison with the notation of the previous section):

$$
u_{n}=2 A_{n} \cos (\omega t) .
$$

Substituting into the equation of motion (2) and balancing the cosine terms leads to the following system of algebraic equations (for $n=1 . . N_{s}$ ),

$$
\left(\omega_{0}^{2}-\omega^{2}\right) A_{n}+3 \xi A_{n}^{3}=\omega_{c}^{2}\left(A_{n+1}+A_{n-1}-2 A_{n}\right),
$$

where $\omega$ is also an unknown.

Substituting the form of mode $N_{s} / 2$ into Eq.(17) (i.e: $A_{n}=(-1)^{n} V$ ) results in a single equation only, which gives the expression of the backbone curve for this mode, i.e. its natural frequency as a function of the amplitude:

$$
\omega_{N_{s} / 2}^{2}=\omega_{0}^{2}+4 \omega_{c}^{2}+3 \xi V^{2} .
$$

In order to compare the NLSE solitons to the HBM solution for mode $N_{s} / 2$, we introduce the continuum approximation into the HBM equation (17), and we assume an out of phase motion (ie $A_{n} \rightarrow(-1)^{n} A_{n}$ ). This leads to the following differential equation:

$$
a^{2} \omega_{c}^{2} \frac{d^{2} A_{n}}{d x^{2}}+\left(\omega_{0}^{2}+4 \omega_{c}^{2}-\omega^{2}\right) A_{n}+3 \xi A_{n}^{3}=0
$$

Using the ansatz $A_{n}=A(n a)=A(x)=V \operatorname{sech}(V \gamma x)$ leads to the following solutions $A(x)$ for the HBM equation:

$$
A(x)=V \operatorname{sech}\left(V \sqrt{\frac{3 \xi}{2 a^{2} \omega_{c}^{2}}} x\right), \quad \text { with } \omega^{2}=\omega_{0}^{2}+4 \omega_{c}^{2}+\frac{3}{2} \xi V^{2},
$$

and finally the HBM solution is given by $u_{n}=2(-1)^{n} A(n a) \cos (\omega t)$. 
Let us now compare the HBM solution in Eq.(19) to the NLSE soliton solution in Eq.(13). In both cases the envelope is given by a hyperbolic secant with the same arguments, i.e the functional forms are the same for both methods. The only difference lies in the expression of the vibration frequency: the NLSE soliton frequency in fact corresponds to an order one Taylor expansion of the HBM frequency, and will therefore have a smaller validity interval.
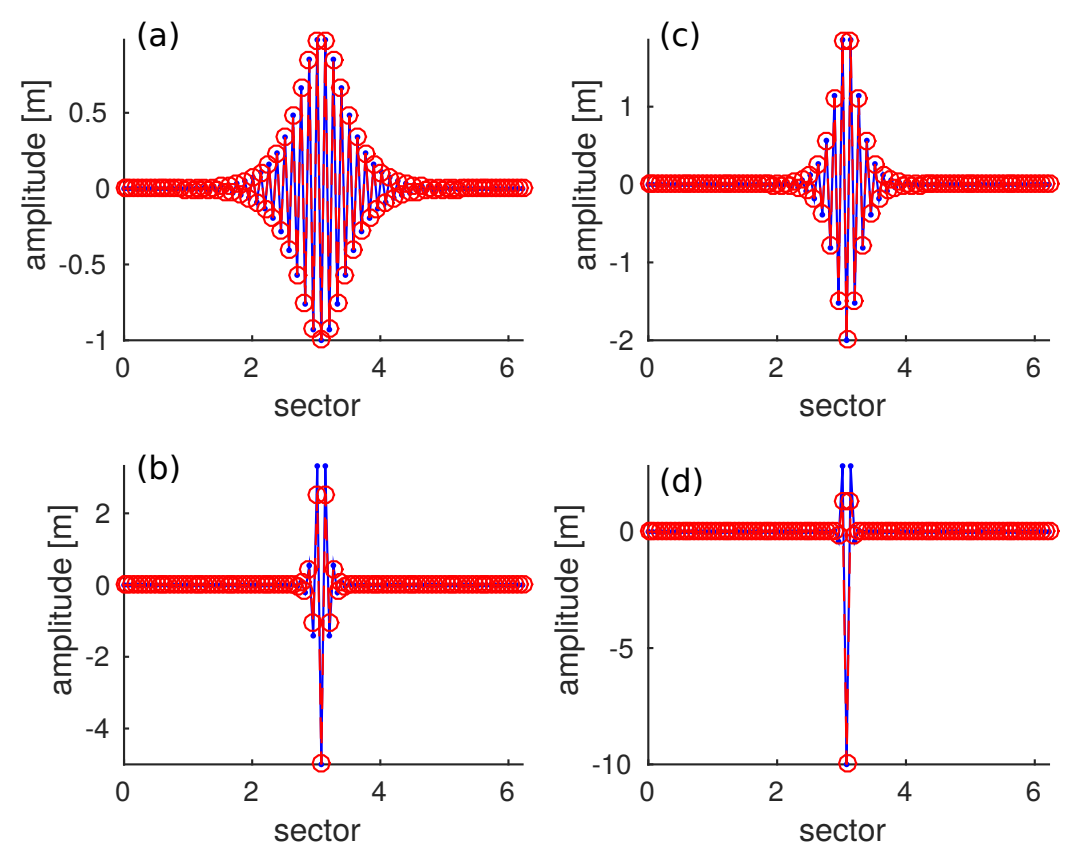

Figure 7: Comparison beetween soliton (.) and HBM (०) solution for different amplitude (from top left to bottom right $\mathrm{V}=1,2,5,10$ ).

Another comparison, between the solution of the continuous equation (19) and the solution of the discrete equation (17), can be made by using the results of Eq.(19) to derive an initial guess for the HBM solution of Eq.(17) for a fixed frequency. This guess is then refined using a Newton-Raphson algorithm. From Fig.7 it can be seen that the "continuous" and "discrete" solutions are indeed very close to each other. Even for high amplitudes, the shape of the HBM solution remains rather well approximated by the shape of the (standing) soliton solution, since, in the worst case (Fig.7, d) the relative difference between their $L_{2}$ norm is smaller than $15 \%$.

Another question is how the spatially localised soliton solutions discussed above are related to the usual normal modes that are not spatially localised. In order to explore potential links of soliton solutions to normal modes, the standing soliton solution obtained by HBM is continued using standard con- 
tinuation procedures with the angular frequency as a parameter. In Fig.8 we plot the backbone curve of the principal non-localised normal mode (blue line) along with the results of the continuation procedure for the solitons (red curve). It turns out that the soliton solution tends to return to and merge with the principal normal mode as amplitude and frequency decrease. Actually, it can be observed that the soliton branch emerges from the principal NNM through a tangent bifurcation, see again [4] for a discussion in the context of physics. The mechanism leading to this bifurcation seems also intuitively accessible: when the soliton amplitude decreases, the packet width increases. For sufficiently low amplitude, the packet does not fit in full into the domain any more, and instead it approaches a more homogeneous shape, as has also been observed above already.

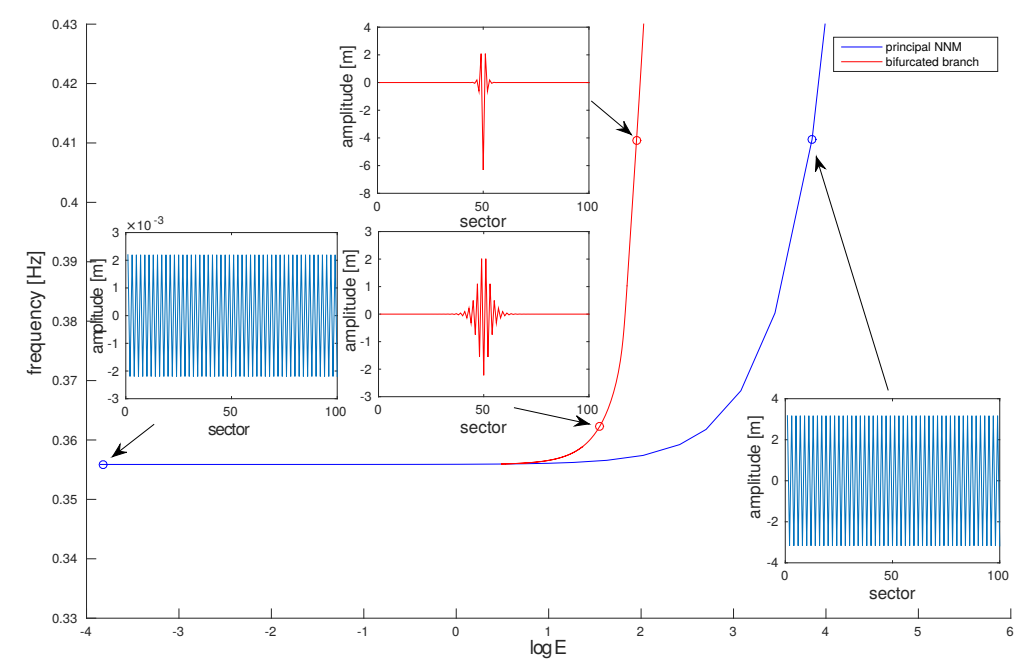

Figure 8: Results of frequency continuation in Frequency-Energy plot. Blue curve: principal non-localised backbone mode $N_{s} / 2$ (Eq.(18)). Red: standing soliton solution (Eq.(20)).

At this point one should note that the behaviour described above has now been seen in a number of physical systems that can be describe through a discrete NLSE, see e.g. an earlier review [4], or more recent findings from nonlinear optics for Kerr type media, [29], where it has been shown that solitons can bifurcate from the upper limit of a Bloch band.

Another interesting aspect, that we would like to touch in brief only, is that the findings and techniques described above open up the possibility to easily obtain vibration states of the system that may be thought to be composed of a number of non-interacting single standing solitons. Indeed, for sufficiently large amplitude, the soliton solutions are strongly localized, up to almost a single 

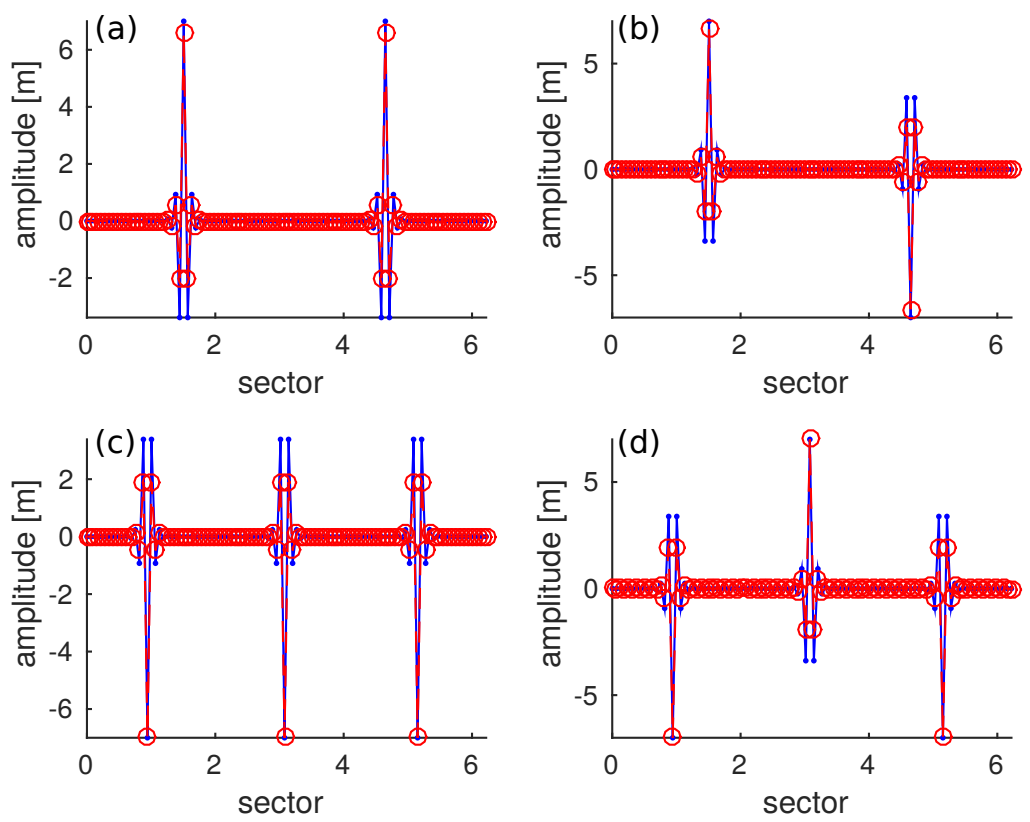

Figure 9: Comparison beetween soliton (.) and HBM (०) solutions for different combination of soliton solution

site. If we introduce another soliton, with the same or a comparable amplitude, localized at another site (sufficiently spaced from the first one), the two solitons do not seem to interact and the combination of those two solitons seems a solution too. Some such soliton combinations are presented in Fig.9, where we again compare the HBM solutions to the soliton solutions. We can see again that HBM solutions and solutions obtained by simulation are very close to each other, and that the combination of solitons actually seems to yield a solution of the HBM system. Basically, almost all combinations can be considered as long as the displacement patterns are sufficiently localized, i.e. the localization sites are sufficiently spaced. This gives an easy way to derive localised and multilocalised solution for mode $N_{s} / 2$. Definitely these numerical findings deserve more detailed study, especially with a view to the multitude of recent work on multi-solitons and higher order solitons $[11,12]$.

\section{Conclusion and Outlook}

This paper studies non-linearly localised vibration states in non-linear cyclic structure composed of coupled duffing oscillators, which can be seen as an approximation for bladed-disk-type structures appearing in turbo-machinery. We 
show that travelling and standing soliton patterns can arise in such systems with the particular feature that the solution becomes more and more localized as the amplitude of motion increases.

In the first part of the paper, we introduce travelling soliton solution. Those solutions correspond to a travelling carrier wave having its amplitude modulated by a travelling envelope. For the hardening type system under study such soliton solutions can only appears for sufficiently high wave numbers. In order to illustrate the existence and properties of travelling soliton solutions, numerical simulations have been carried out for an example system composed of $N_{s}=100$ sectors, and results are compared with a linearised system.

The second part of the paper focuses on the limiting case where the group velocity approaches zero. In this case the soliton solution does not travel any more and corresponds to a spatially stationary purely time periodic solution. For this limiting case, solitary solutions can be determined through two ways: (i) integration of the fully non-linear evolution equations in time, starting with initial conditions derived from the analytic solution for envelope solitons of the NLSE, and (ii) by HBM. In this case, the main advantage of the HBM, is that it is able to derive an expression for standing solutions without any assumption on the order of magnitude of the non-linearity. An interesting feature is that the standing solitons merge with the well known backbone curves of the nonlocalised normal vibration modes for small amplitudes through bifurcation.

We expect the present study on the existence of envelope solitons in cyclic systems to open new avenues of investigation in the turbomachinery community. Here only, a simple model considering a single degree of freedom per sector was considered. Further study should consider the case of several dof per sector, the ultimate goal being to consider a full structure by using finite element models. Different king of non-linearity should also be considered (hyper-elasticity, geometric non-linearity, blade casing interaction, ...). Also the interaction of the non-linear structural dynamics with the surrounding non-linear flow will need consideration, to better understand the dynamical phenomena potentially arising in turbo-machinery components like gas turbines or aero-engines. Another field of future work will be numerical methods. As already noted, solitary waves might be regarded as a special class of non-linear normal modes, and further study to show the relationship between the non-localised and the localised non-linear normal modes seems worth-while and required too. The development of efficient numerical tools for these purposes, such as a multiple scale HBM, will also be a topic of future study.

\section{A derivation of soliton-like solution}

In this appendix, we show how the Schrodinger equation can be derived for our system. Following Remoissonet [18], we search for a solution in the form of a 
carrier wave modulated by an envelope which varies slowly in time and space,

$$
u_{n}=\Psi_{n}(X, T) e^{i\left(k(n-1) a-w_{k} t\right)}+c . c .=2 \operatorname{Re}\left(\Psi_{n}(X, T) e^{i\left(k(n-1) a-w_{k} t\right)}\right),
$$

where $a$ is the sector parameter $\left(a=\frac{2 \pi}{N_{s}}\right), X=\epsilon x$ and $T=\epsilon t$. Computing the time derivative leads to:

$$
\ddot{u}_{n}=\left(-\omega_{k}^{2} \Psi_{n}-2 i \epsilon \omega_{k} \frac{d \Psi}{d T}+\epsilon^{2} \frac{d^{2} \Psi}{d T^{2}}\right) e^{i\left(k(n-1) a-w_{k} t\right)}
$$

We also make use of the continuum approximation $(a<<1)$ so that the amplitude of the next sector can be expressed using Taylor expansion :

$$
\Psi_{n \pm 1}=\Psi(n a \pm a)=\Psi_{n} \pm a \frac{d \Psi_{n}}{d x}+\frac{a^{2}}{2} \frac{d^{2} \Psi_{n}}{d x^{2}}+\text { H.O.T }
$$

Substituting Eq.(22) and (23) in the equation of motion (2), assuming that the non-linear term is of order $\epsilon^{2}$ and keeping only the first harmonics leads to:

$$
\begin{array}{r}
-\omega_{k}^{2} \Psi_{n}-2 i \epsilon \omega_{k} \frac{d \Psi}{d T}+\epsilon^{2} \frac{d^{2} \Psi}{d T^{2}}+\left(\omega_{0}^{2}+2 \omega_{c}^{2}(1-\cos (k a))\right) \Psi_{n}+3 \epsilon^{2} \xi|\Psi|^{2} \Psi= \\
2 i \epsilon a \omega_{c}^{2} \sin (k a) \frac{d \Psi}{d X}+2 \epsilon^{2} \frac{a^{2} \omega_{c}^{2}}{2} \cos (k a) \frac{d^{2} \Psi}{d X^{2}}
\end{array}
$$

Using the linear relationship in Eq.(4) for the eigen-frequencies, the previous equation simplifies to

$$
i \epsilon\left(\frac{d \Psi}{d T}+c_{g} \frac{d \Psi}{d X}\right)+\epsilon^{2} \frac{1}{2 \omega_{k}}\left(\Lambda \frac{d^{2} \Psi}{d X^{2}}-\frac{d^{2} \Psi}{d T^{2}}\right)-\epsilon^{2} \Xi|\Psi|^{2} \Psi=0,
$$

where

$$
c_{g}=\frac{a \omega_{c}^{2}}{\omega_{k}} \sin (k a), \Lambda=a^{2} \omega_{c}^{2} \cos (k a), \Xi=\frac{3 \xi}{2 \omega_{k}} .
$$

We now introduce the change of variables $\eta=X-c_{g} T, \tau=\epsilon T$ to change into a coordinate system moving with group velocity. In this case we have

$$
\frac{d}{d X}=\frac{d}{d \eta}, \frac{d}{d T}=c_{g} \frac{d}{d \eta}+\epsilon \frac{d}{d \tau}, \frac{d^{2}}{d T}=c_{g}^{2} \frac{d^{2}}{d \eta^{2}}+2 c_{g} \epsilon \frac{d^{2}}{d \tau d \eta}+\epsilon^{2} \frac{d^{2}}{d \tau^{2}} .
$$

Substituting the previous relations in Eq.(25) and keeping only terms of order $\epsilon^{2}$ leads to

$$
i \frac{d \Psi}{d \tau}+\frac{1}{2 \omega_{k}}\left(\Lambda-c_{g}^{2}\right) \frac{d^{2}}{d \eta^{2}}-\Xi|\Psi|^{2} \Psi=0 .
$$

Using the definition of the group velocity dispersion $\frac{d^{2} w_{k}}{d k^{2}}=\frac{1}{\omega_{k}}\left(\Lambda-c_{g}^{2}\right)$ results in

$$
i \frac{d \Psi}{d \tau}+\frac{1}{2} \frac{d^{2} w_{k}}{d k^{2}} \frac{d^{2} \Psi}{d \eta^{2}}-\Xi|\Psi|^{2} \Psi=0,
$$

which corresponds to the standard scaled form of the non-linear Schrodinger equation,

$$
i \frac{d \Psi}{d \tau}+\alpha \frac{d^{2} \Psi}{d \eta^{2}}+\beta|\Psi|^{2} \Psi=0,
$$

with $\alpha=\frac{1}{2} \frac{d^{2} w_{k}}{d k^{2}}, \beta=-\Xi$. 


\section{References}

[1] M.A. Davies and F.C. Moon. Solitons, chaos and modal interaction in periodic structure. Nonlinear Dynamics: The Richard Rand 50th Anniversary Volume: Series on Stability, Vibration and Control of Systems, Series B, 2:119-143, 1997.

[2] D.J. Ewins. The effect of mistuning upon the forced vibrations of bladed disks. Journal of Sound and Vibration, 9(1):65-79, 1969.

[3] M. Feldman. Hilbert transform in vibration analysis. Mechanical System and Signal Processing, 25:735-802, 2011.

[4] S. Flach and A.V. Gorbach. Discrete breathers advances in theory and applications. Physics Reports, 467(13):1 - 116, 2008.

[5] F. Georgiades, M. Peeters, G. Kerschen, and J.C. Golinval. Modal analysis of a nonlinear periodic structure with cyclic symmetry. AIAA journal, 47(4):21014-1025, 2009.

[6] A. Grolet and F. Thouverez. Free and forced vibration analysis of a nonlinear system with cyclic symmetry: Application to a simplified model. Journal of Sound and Vibration, 331(12):2911-2928, 2012.

[7] A. Grolet and F. Thouverez. Computing multiple periodic solutions of nonlinear vibration problems using the harmonic balance method and Groebner bases. Mechanical Systems and Signal Processing, 52:529-547, February 2015 .

[8] N. Karjanto and E. Van Groesen. Derivation of the nls breather solutions using displaced phase amplitude variables. In 5th SEAMS-GMU International Conference on Mathematics and its Applications, pages 43-50, 2007.

[9] V. V. Konotop. Small emplitude envelope soliton in nonlinear lattices. Wiley Interscience, 1996.

[10] A. Lagendijk, B. Van Tiggelen, and D. Wiersma. Fifty years of anderson localization. Physics Today, 62(8):24-29, 2009.

[11] Y. Martel and F. Merle. Multi solitary waves for nonlinear schrdinger equations. Annales de l'Institut Henri Poincare (C) Non Linear Analysis, 23(6):849 - 864, 2006.

[12] N. D. Nguyen and Nguyen B. Generation of high order multi-bound solitons and propagation in optical fibers. Optics Communications, 282(12):2394 $2406,2009$.

[13] E.P. Petrov and D.J. Ewins. Analysis of the worst mistuning patterns in bladed disk assemblies. Journal of Turbo-machinery, transaction of the ASME, 125:623-631, 2003. 
[14] G.V, Dreiden, A.M. Samsonov, I.V. Semenova and A.G. Shvartz. Bulk strain soliton in a cylindrical shell. Proceedings of the international conference day on diffraction, 69-75, 2014.

[15] A.M. Samsonov. Strain solitons in solids and how to construct them. Monographs and surveys in pure and applied mathematics, 117, 2001.

[16] D. Boulahbal and S.H. Crandall. Self excited harmonic ans solitary waves i na spinning disk. VIB-4093, Proceedings of DETC 97, September 14-17, Sacramento, CA, 1997.

[17] G. Maugin. Solitons in elastic solids (1938-2010). Mechanics research communications,38:341-349, 2011.

[18] M. Remoissonet. Waves called solitons. Springer, 1994.

[19] M. Rosenberg, R. The normal modes of nonlinear n-degree of freedom systems. Journal of Applied Mechanics, 29(1):7-14, 1962.

[20] M. Rosenberg, R. On nonlinear vibrations of systems with many degree of freedom systems. Journal of Applied Mechanics, 9(1):155-242, 1966.

[21] E. Sarrouy, A. Grolet, and F. Thouverez. global and bifurcation analysis of a structure with cyclic symmetry. International journal of nonlinear mechanics, 46(5):727-737, 2011.

[22] W. Shaw, S. and C. Pierre. Nonlinear normal modes and invariant manifolds. Journal of Sound and Vibration, 150:170-173, 1991.

[23] W. Shaw, S. and C. Pierre. Normal modes for nonlinear vibratory systems. Journal of Sound and Vibration, 164:85-124, 1993.

[24] Y. Starosvetsky and A. F. Vakakis. Traveling waves and localized modes in one-dimensional homogeneous granular chains with no precompression. Phys. Rev. E, 82:026603, Aug 2010.

[25] S. Takeno. Theory of stationary anharmonic localized modes in solids. Journal of the physical society of Japan, 61(8):2821-2834, 1992.

[26] A.F. Vakakis. Normal modes and localization in non-linear systems. WileyInterscience publication, 1996.

[27] G. Von Groll and D.J. Ewins. The harmonic balance method with arclength continuation in rotor/stator contact problem. Journal of Sound and Vibration, 241(2):223 - 233, 2001.

[28] Y.J. Yana, P. Cuia, and H. Hao. Vibration mechanism of a mistuned bladed disk. Journal of Sound and Vibration, 317:294-307, 2008.

[29] J. Yang. Nonlinear Waves in integrable and non integrable systems. SIAM, 2010. 